\title{
Détermination de la puberté et de l'âge à la première mise-bas des chevrettes en élevage familial à Lubumbashi, République Démocratique du Congo.
}

\author{
Mayeriya $\mathrm{K}^{1}$, Ngona $\mathrm{IA}^{2}$, Mbiya $\mathrm{L}^{1}$, Khang'Maté $\mathrm{AB}^{2}$ \\ ${ }^{1}$ Département de Zootechnie, Faculté de Sciences Agronomiques, Université de Lubumbashi, RDC. \\ ${ }^{2}$ Service de Reproduction, Obstétrique et Insémination Artificielle, Faculté de Médecine Vétérinaire, Université de \\ Lubumbashi, RDC. \\ *ingona@yahoo.fr,
}

Original submitted in on $20^{\text {th }}$ October 2016. Published online at www.m.elewa.orgon $31^{\text {st }}$ January 2017 http://dx.doi.org/10.4314/jab.v109i1.11

\section{RESUME}

Objectif : L'objectif de l'étude consistait à déterminer la puberté des caprins, préciser l'âge à la $1^{\text {re }}$ mise-bas et à assurer une bonne gestion de la reproduction de la chèvre à Lubumbashi. Les investigations, menées de décembre 2010 à mai 2011, s'étaient appuyées sur 25 chevrettes de race locale ayant fait l'objet de caractérisation zootechnique.

Méthodologie et Résultats : Le suivi de la reproduction s'était effectué par des visites mensuelles. Le constat de gestation par échographie des femelles sélectionnées, était effectué à une fréquence trimestrielle. Les données étaient traitées par la comparaison des différents axes d'élevage par rapport à leurs coefficients de corrélation en mesurant le niveau de liaison entre deux variables corrélatives telles que le poids et la taille d'un individu. Les résultats consistaient en une mise en évidence d'une disproportion entre les femelles gestantes et non gestantes (21 vs $79 \%)$. Les résultats observés présentaient des élevages avec plus de femelles à 8 dents de lait par rapport à celles à 2 dents adultes (60 vs 40\%). Le poids et la taille au garrot des individus à 8 dents de lait $(9,9-18 \mathrm{~kg}$ et $43,5-49,5 \mathrm{~cm})$ et à 2 dents d'adultes (14,8-22,4 kg et 49,1-52,0 cm) avaient été précisés pour les différents axes d'élevage. Il en était de même des chevrettes gestantes (20,8-31,6\%) dont le poids variait de 8,8 à $18,95 \mathrm{~kg}$.

Conclusions et application des résultats : Les individus dont l'âge correspondait à 8 dents de lait avaient présenté une proportion nulle de mise-bas. A un âge correspondant à 2 dents d'adultes, les proportions de mise-bas correspondaient à 30,8\%. Les mise-bas observées chez les individus à 2 dents d'adultes permettaient de déterminer le moment de puberté à 10 mois. En perspectives, il serait utile de déterminer les facteurs d'influence d'une puberté tardive, notamment l'environnement alimentaire. En effet, une bonne gestion alimentaire permettra à l'éleveur de pallier le caractère tardif de la puberté, un des facteurs responsables de l'absence de rentabilité de cet élevage familial. L'atteinte d'une puberté précoce est un atout pour une productivité numérique au travers des mise-bas.

Mots-clés : chevrettes, poids et taille, gestation, âge à la $1^{\text {re }}$ mise-bas, puberté, élevage périurbain, Lubumbashi

\footnotetext{
ABSTRACT

Objective: The objective of the study was to determine caprine puberty, specify age at 1 st farrowing and ensure good management of goat breeding in Lubumbashi. Investigations, conducted from December 2010 to May 2011, were based on 25 young local breeds that had been zootechnically characterized.
} 
Methodology and Results: Monitoring of breeding was done by monthly visits. The ultrasound gestation of selected females was performed on a quarterly basis. Data were processed by comparing different rearing axes with respect to their correlation coefficients by measuring the level of linkage between two correlative variables such as weight and height of an individual. Results consisted of a disproportion between pregnant and non-pregnant females (21 vs $79 \%$ ). Results observed showed farms with more females with 8-teeth milk than those with 2-adult teeth (60 vs 40\%). The weight and height at the withers of 8-teeth milk (9.9-18 $\mathrm{kg}$ and $43.5-49.5 \mathrm{~cm})$ and 2-adult teeth $(14.8-22.4 \mathrm{~kg}$ and $49.1-52.0 \mathrm{~cm})$ were specified for different rearing axes. The same was true for pregnant goats (20.8-31.6\%), whose weight varied from 8.8 to $18.95 \mathrm{~kg}$.

Conclusion and application of findings: Individuals whose age was 8-teeth milk had a zero proportion of farrowing. At an age corresponding to 2-adult teeth, the farrowing proportions corresponded to $30.8 \%$. Feedings observed in adults with 2-adult teeth were used to determine puberty at 10 months. In perspective, it would be useful to determine influencing factors of late puberty, including food environment. Indeed, good food management will allow the breeder to palliate the tardiness of puberty, one of the factors responsible for the lack of profitability of this family breeding. Achieving early puberty is an asset for digital productivity through deliveries.

Key-words: young female caprine, weight and height, gestation, age at 1st birth, puberty, peri-urban farming, Lubumbashi

\section{INTRODUCTION}

La puberté d'une femelle correspond au moment de la première ovulation (Sakural et al., 2004). Elle résulte d'une série d'événements complexe du développement qui surviennent au niveau de l'axe hypothalamo-hypophysaire (Gordon, 2004). L'âge à la puberté ou l'apparition du premier œstrus est sensiblement variable en fonction des facteurs génétiques, de la saison de naissance et de la conduite de l'élevage ou de leur interaction (Freitas et al., 2004). Chez les caprins de race locale des zones tropicale et subtropicale, la puberté apparait, en général, entre 8 et 14 mois. Chez les races européennes importées dans les zones tropicales, la puberté commence plus tardivement que chez les animaux locaux (Delgadillo et al., 1997). Sous les tropiques, l'âge à la première mise-bas est entre 18 et 26 mois dans la plupart

\section{MATERIEL ET METHODES}

Milieu: Nos investigations ont eu lieu en milieu périurbain de Lubumbashi, auprès des élevages familiaux de caprins de décembre 2010 à mai 2011. Ce milieu présentait une saison sèche (avril-octobre) et une saison pluvieuse (novembre-mars). Le suivi d'élevage avait permis de mettre en évidence 11 éleveurs et dont les effectifs respectifs en début des investigations étaient de 25 chevrettes appartenant à l'environnement spécifique à la race naine africaine et mieux identifiées comme des caprins de race locale (Fig.1). des cas (Le Gal \& Planchenault, 1993). Un retard de puberté relève d'une croissance lente des animaux associée aux conditions tropicales (Freitas et al., 2004 ; Zarazaga et al., 2005). La relation entre le statut nutritionnel, la croissance corporelle et l'âge à la puberté est mieux exprimée par la sous alimentation qui peut retarder la puberté jusqu'à une année (Gordon, 2004 ; Sakurai et al., 2004). Une conduite optimale des chevrettes pendant la période d'élevage, doit permettre une première mise-bas à l'âge de 12 à 13 mois. La durée de gestation est de 5 mois. L'objectif de l'étude consistait à déterminer la puberté des caprins, préciser l'âge à la 1 re mise-bas et à assurer une bonne gestion de la reproduction de la chèvre en élevage familial à Lubumbashi.

\section{Matériel et Méthodes \\ Identification des individus : L'identification reposait sur des boucles d'oreilles (Fig.2). Des données zoométriques à savoir la taille au garrot et le poids vif étaient prélevées mensuellement. La taille au garrot était prélevée à l'aide de mètre ruban. Le poids vif était le résultat d'un double pesé à l'aide d'une balance pèse-personne pour déduire le poids vif de l'animal. Quoique l'approche soit subjective, l'âge découlait de l'observation de la dentition (Ngona, 2015).}




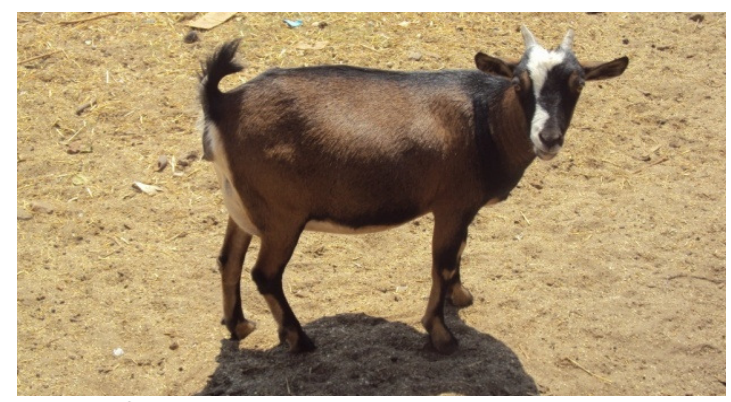

Fig. 1 Caprin de race locale

Suivi de la reproduction: Au cours des visites mensuelles, la surveillance des chevrettes permettait d'enregistrer les données de mise-bas. Le constat de gestation par échographie était effectué à une fréquence trimestrielle (Fig. 3). Les femelles examinées

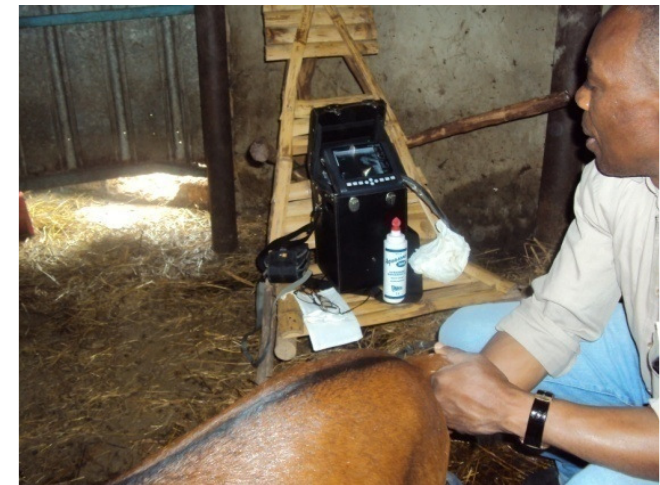

Fig.3 : Constat de gestation par échographie

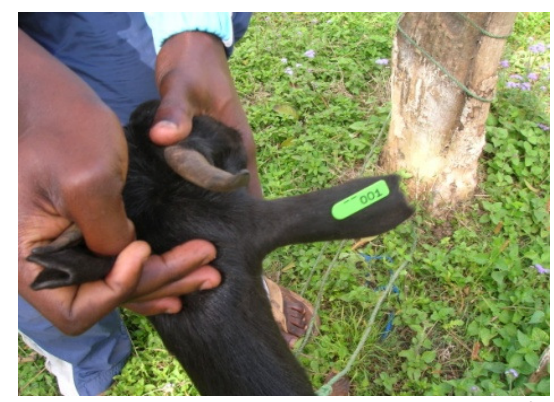

Fig. 2 Boucles d'oreille pour l'identification

étaient gravides ou non gravides selon que l'échographie montrait la présence ou l'absence de l'embryon ou du fœtus. L'observation des placentomes était le signe le plus courant qui traduisait la présence du fœtus (Fig. 4).

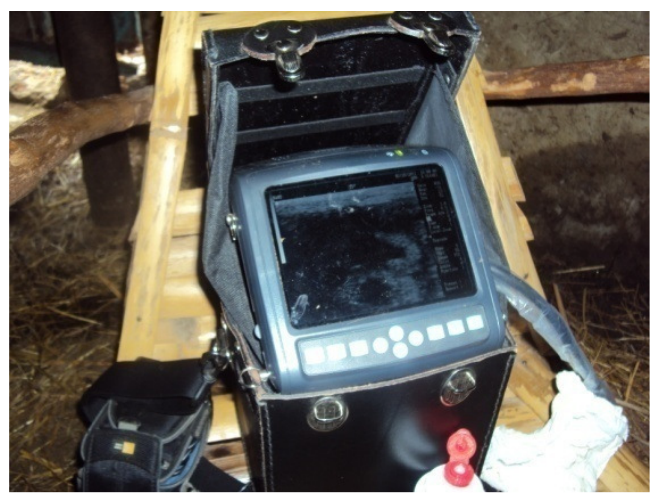

Fig. 4 : Présence de placentomes

de liaison entre deux variables corrélatives telles que le poids et la taille d'un individu.

n'avaient pas respectivement d'individus avec 8 dents de lait et 2 dents d'adultes. Les boucs correspondaient à une proportion de 7,52\% par rapport aux femelles adultes. L'absence de boucs était observée dans $62,5 \%$ d'élevages. vs $40 \%$ ) (Tableau 1). Cependant, $25 \%$ d'élevages

Tableau 1: Age des chevrettes sélectionnées

\begin{tabular}{l|l|l|l|l}
\hline Axe d'élevage & élevage & 8 dl & 2DA & total \\
\hline Kafubu & $\begin{array}{l}\text { naviundu } \\
\text { tshilombo } \\
\text { Kampemba }\end{array}$ & 2 & 1 & 3 \\
& $\begin{array}{l}\text { clementine } \\
\text { mukendi }\end{array}$ & 3 & 2 & 4 \\
Ruashi & & & 3 \\
pasteur & 2 & 2 & 2 \\
Karavia & golf & & 2 \\
Katuba & bilonda & 5 & 3 & 3 \\
Total & mutonkole & 1 & 1 & 6 \\
& $(\mathrm{n})$ & 1 & 2 \\
\hline & $(\%)$ & 15 & 10 & 25 \\
& 60 & 40 & 100 \\
\hline
\end{tabular}

Légende : $8 \mathrm{dl}-8$ dents de lait, 2DA - 2 dents d'adultes 
Détermination du poids et de la croissance : L'étude descriptive avait précisé le poids et la taille pour les deux catégories d'âge des femelles, à savoir à 8 dents de lait $(9,9-18 \mathrm{~kg}$ et $43,5-49,5 \mathrm{~cm})$ et à 2 dents d'adultes (14,8-22,4 kg et 49,1-51,6 cm) (Tableau 2). L'étude relationnelle avait présenté un coefficient de corrélation qui traduisait l'importance du lien entre le poids et la taille des individus à 8 dents de lait $(0,23-$ $0,58)$ et à 2 dents d'adultes $(0,18-0,86)$. Un coefficient de corrélation inférieur à 0,5 traduisait un faible lien entre ces deux paramètres. En effet, pour $r<0,5$, le poids de l'individu ne correspondait pas à sa taille (Tableau 3).

Tableau 2 : Poids $(\mathrm{kg})$ et taille $(\mathrm{cm})$ des chevrettes selon les axes d'élevage

\begin{tabular}{l|l|l|l|l}
\hline \multirow{2}{*}{ Axe d'élevage } & \multicolumn{2}{l|}{ chevrettes à 8dl } & \multicolumn{2}{l}{ chevrettes à 2DA } \\
\cline { 2 - 5 } & poids & taille & poids & taille \\
\hline Kafubu & 10,8 & 47,1 & 14,8 & 50,0 \\
Kampemba & 9,9 & 43,5 & 19,0 & 51,3 \\
Karavia & 10,0 & 45,7 & 22,4 & 51,6 \\
Ruashi & 18,0 & 49,5 & 17,2 & 49,1 \\
Golf & - & - & 17,3 & 50,8 \\
Katuba & - & - & 17,0 & 52,0 \\
\hline
\end{tabular}

Légende : $8 \mathrm{dl}-8$ dents de lait, 2DA - 2 dents d'adultes

Tableau 3 : Coefficient de corrélation des paramètres poids et taille des chevrettes selon les axes d'élevage

\begin{tabular}{l|l|l}
\hline Axe d'élevage & chevrettes à 8dl & chevrettes à 2DA \\
\hline Kafubu & 0,58 & 0,27 \\
Kampemba & 0,36 & 0,27 \\
Karavia & 0,23 & 0,48 \\
Ruashi & 0,58 & 0,86 \\
Golf & - & 0,52 \\
Katuba & - & 0,18 \\
\hline
\end{tabular}

Légende : $8 \mathrm{dl}-8$ dents de lait, 2DA - 2 dents d'adultes

Détermination du taux de gestation des chevrettes : Le constat de gestation périodique présentait des proportions de femelles gestantes de 31,6\% (décembre) et de $20,8 \%$ (février). Le poids des chevrettes variait de 8,8 à $17,95 \mathrm{~kg}$ (décembre) et de 9,63 à 18,95 kg (février). Cependant, les femelles gestantes avaient un poids compris entre 11,26 et 16,9 $\mathrm{kg}$ (décembre) et 17,4 et 18,95 kg (février) (Tableau 4).

Tableau 4 : Constat de gestation des chevrettes au cours du suivi d'élevages

\begin{tabular}{l|c|c|c|c|c|c|c|c}
\hline \multirow{2}{*}{ Élevage } & & \multicolumn{3}{|c|}{ décembre } & & \multicolumn{3}{c}{ février } \\
\cline { 2 - 9 } & $\mathbf{n}$ total & $\mathbf{n}$ & $\%$ & poids moyen & $\mathbf{n}$ total & $\mathbf{n}$ & $\%$ & poids moyen \\
\hline Cliniq vét & 2 & 0 & 0 & 17,95 & 2 & 0 & 0 & 17,15 \\
Naviundu & 3 & 0 & 0 & 12,40 & 3 & 1 & 33 & 17,40 \\
Tshilombo & 2 & 0 & 0 & 13,10 & 6 & 0 & 0 & 11,18 \\
Mukendi & 2 & 2 & 100 & 16,70 & 2 & 1 & 50 & 18,50 \\
Clementine & 1 & 0 & 0 & 8,80 & 3 & 0 & 0 & 9,63 \\
Bilonda & 5 & 2 & 40 & 11,26 & 4 & 2 & 50 & 18,0 \\
Mutonkole & 2 & 1 & 50 & 16,70 & 2 & 0 & 0 & 15,50 \\
Pasteur & 2 & 1 & 50 & 16,90 & 2 & 1 & 50 & 18,95 \\
Total & 19 & 6 & 31,6 & & 24 & 5 & 20,8 & \\
\hline
\end{tabular}

Détermination de l'âge à la première mise-bas : L'étude avait montré une proportion nulle de mise-bas chez les individus dont l'âge correspondait à 8 dents de lait. A un âge correspondant à 2 dents d'adultes, la proportion de mise-bas correspondait à 30,8\% (4/14 femelles) (Tableau 5) 
Tableau 5 : Détermination de l'âge à la $1^{1}$ re mise-bas

\begin{tabular}{|c|c|c|c|c|c|c|}
\hline \multirow[t]{3}{*}{ Élevage } & \multicolumn{6}{|c|}{ Age à la $1^{\text {re }}$ mise-bas } \\
\hline & \multicolumn{3}{|c|}{$8 \mathrm{dl}$} & \multicolumn{3}{|c|}{ 2DA } \\
\hline & $\mathrm{n}$ total & $\mathrm{n}$ & $\%$ & $\mathrm{n}$ total & $n$ & $\%$ \\
\hline Cliniq vét & 3 & 0 & 0 & 0 & 0 & 0 \\
\hline Naviundu & 0 & 0 & 0 & 3 & 1 & 33 \\
\hline Tshilombo & 4 & 0 & 0 & 2 & 0 & 0 \\
\hline Mukendi & 0 & 0 & 0 & 2 & 1 & 50 \\
\hline Clementine & 3 & 0 & 0 & 0 & 0 & 0 \\
\hline Bilonda & 3 & 0 & 0 & 2 & 0 & 0 \\
\hline Mutonkole & 0 & 0 & 0 & 2 & 1 & 50 \\
\hline Pasteur & 0 & 0 & 0 & 2 & 1 & 50 \\
\hline Total & 13 & 0 & 0 & 13 & 4 & 30,8 \\
\hline
\end{tabular}

Légende : $8 \mathrm{dl}-8$ dents de lait, 2DA - 2 dents d'adultes

\section{DISCUSSION}

Chevrettes sélectionnées : Au terme de la sélection, il était mis en évidence une disproportion entre les femelles gestantes et celles non gestantes (21 vs $79 \%$ ). Cette disproportion n'était pas compatible avec les objectifs de la gestion de la reproduction. Le troupeau devait présenter plus de $60 \%$ des femelles gestantes. Les résultats observés présentaient des élevages avec plus de femelles à 8 dents de lait par rapport à celles à 2 dents d'adultes (60 vs 40\%) (Tableau 1). Une bonne productivité numérique et pondérale, de même que des performances de reproduction satisfaisantes reposent sur l'élevage des chevrettes.

Détermination du poids et de la croissance : Les paramètres poids et taille pour les deux catégories d'âge des chevrettes, à savoir à 8 dents de lait (9,9-18 $\mathrm{kg}$ et 43,5-49,5 cm) et à 2 dents d'adultes (14,8-22,4 $\mathrm{kg}$ et 49,1-51,6 cm) (Tableau 2) étaient, respectivement, faibles aux résultats obtenus chez la chèvre à Lubumbashi $(20,5 \mathrm{~kg}$ et $53,2 \mathrm{~cm}$ ) (Ngona, 2015). Le coefficient de corrélation traduisait l'importance du lien entre le poids et la taille avec des fortes variations chez les individus à 8 dents de lait $(0,23-0,58)$ et à 2 dents d'adultes $(0,18-0,86)$ (Tableau 3). Le forte ou la faible corrélation permettait d'apprécier la conduite d'élevage qui pourrait justifier la puberté précoce ou tardive. Cette dernière est un facteur d'absence de rentabilité dans un élevage. Les deux paramètres (poids et taille) permettaient de définir le moment de puberté. En effet, l'atteinte de la puberté était liée à l'acquisition d'un poids critique. Des travaux chez la chèvre locale avaient montré une corrélation entre le poids et la taille au garrot (Ngona, 2015). Les chevrettes atteignaient la puberté et pouvaient être prête à la saillie vers 6 à 8 mois. Par contre les chevrettes ne devraient pas être saillies avant d'atteindre 60 à $75 \%$ du poids de l'adulte pour éviter le ralentissement de la croissance. Un retard de puberté relevait d'une croissance lente des animaux associée aux conditions tropicales, (Greyling, 2000 ; Freitas et al., 2004). L'âge à la puberté était mieux exprimé par la sous alimentation qui pouvait retarder la puberté jusqu'à une année (Gordon, 2004). A cet effet, sous les tropiques, la précarité des aliments en saison sèche, a une conséquence sur la croissance des chevrettes. Les caprins gagnent plus de poids en saison de pluie à cause de l'abondance des aliments ou fourrage. Ce gain pondéral est favorable à l'apparition de la puberté (Le Gal \& Planchenault, 1993).

Détermination du taux de gestation des chevrettes : La proportion de gestation observée était de 20,8$31,6 \%$ chez des chevrettes dont le poids variait de 11,26 à 18,95 kg (Tableau 4). La gestation était la conséquence de saillie fécondante. Cette dernière n'était possible que si les femelles manifestaient un comportement œstral. Selon Delgadillo et al. (1997), $50 \%$ des premiers œstrus détecté n'étaient pas accompagnés d'ovulation et $36 \%$ des premières ovulations n'étaient pas accompagnés de comportement œstral. En gestion de la reproduction, 1 bouc convient pour 25-30 femelles en monte libre. L'absence de bouc dans quelques élevages est une contrainte non négligeable.

Détermination de l'âge à la $1^{\text {re }}$ mise-bas: Une proportion nulle de mise-bas était observée chez les individus dont l'âge correspondait à 8 dents de lait. A un âge correspondant à 2 dents d'adultes, les proportions de mise-bas étaient de 30,8\% (Tableau V). En milieu tropical, l'âge moyen au 1 er chevrotage ou l'intervalle naissance-chevrotage varie entre 543 et 780 jours. Le génotype et la croissance influencent ce paramètre (Das \& Sendalo, 1993; Le Gal \& Planchenault, 1993). En effet, ces résultats correspondaient à une saillie fécondante à 21 mois, soit des individus à 4 dents d'adultes. Les chevrettes présentaient de mise-bas à 2 dents d'adultes correspondant à 15 mois d'âge. Ceci traduisait une saillie fécondante à 10 mois. Cette valeur 
permettait de déterminer le moment de puberté à 10 mois. Des travaux antérieurs avaient montré que la puberté était atteinte à 6-8 mois. L'âge à la puberté ne correspondait pas à l'âge de mise à la reproduction (Webb \& Mamabolo, 2004). Ceci était lié au poids de la

\section{CONCLUSION ET APPLICATION DES RESULTATS}

L'étude avait mis en évidence une disproportion entre les femelles gestantes et celles non gestantes (21 vs $79 \%$ ). Les résultats observés présentaient des élevages avec plus de femelles à 8 dents de lait par rapport à celles à 2 dents d'adultes (60 vs $40 \%$ ). Le poids et la taille pour les individus à 8 dents de lait $(9,9-18 \mathrm{~kg}$ et $43,5-49,5 \mathrm{~cm}$ ) et pour les individus à 2 dents d'adultes $(14,8-22,4 \mathrm{~kg}$ et 49,1-52,0 $\mathrm{cm})$ avaient été précisés pour les différents axes. II en était de même des chevrettes gestantes $(20,8-31,6 \%)$ dont le poids variait de 11,26 à $18,95 \mathrm{~kg}$. Les individus dont l'âge correspondait à 8 dents de lait avaient présenté une proportion nulle de mise-bas. A un âge correspondant à 2 dents d'adultes, les proportions de mise-bas

\section{REMERCIEMENTS}

Nos remerciements sont adressés au PIC Cabrilu (Projet Inter Universitaire Ciblé, Université de Liège et Université de Lubumbashi) qui a supporté les frais de carburant pour le déplacement au cours du suivi d'élevages. Aussi, les éleveurs sont remerciés pour leur convivialité au cours du suivi des élevages. Enfin, la jeune femelle (Le Gal \& Planchenault, 1993). L'aspect poids critique observé chez les individus à 8 dents de lait (Tableau III) était un facteur d'influence de la proportion nulle de mise-bas relevée chez ces individus.

correspondaient à $30,8 \%$. Les mise-bas observées chez les individus à 2 dents d'adultes correspondant à 15 mois d'âge traduisaient une saillie fécondante à 10 mois. Cette valeur permettait de déterminer le moment de puberté à 10 mois. En perspectives, il serait utile de déterminer les facteurs d'influence d'une puberté tardive, notamment l'environnement alimentaire. En effet, une bonne gestion alimentaire permettra à l'éleveur de pallier le caractère tardif de la puberté, un des facteurs responsables de l'absence de rentabilité de cet élevage familial. L'atteinte d'une puberté précoce est un atout pour une productivité numérique au travers des mise-bas.

collaboration avec l'Université de Liège au travers du Service de Thériogénologie des animaux de production de la Faculté de Médecine Vétérinaire a permis d'obtenir un financement de la CUD (Commission Universitaire pour le Développement /Belgique) pour l'achat d'un échographe.

\section{BIBLIOGRAPHIE}

Das SM and Sendalo DS, 1993. Comparative performance of improved meat goats in Malya, Tanzania. . In : Lebbie SHB, Rey B, Irungu EK (Eds), Small Ruminant Research and Development in Africa: Proceedings of the second Biennial Conference of the African Small Ruminant Research Network, AICC, Arusha, Tanzania, 7-11 December 1992. ILCA (International Livestock Centre for Africa)/ CTA (Technical Centre for Agricultural and Rural Cooperation). ILCA, Addis Ababa, Ethiopia, 268 pp.

Delgadillo JA, Malpaux B, Chemineau P, 1997. La reproduction des caprins dans les zones tropicales et subtropicales. INRA Productions Animales 10: 33-41.

Freitas VJF, Rondina D, Nogueira DM, Simplicio AA, 2004. Postpartum anoestrus in Anglo-Nubian and Saanen goats raised in the semi-arid of North-eastern Brazil. Small Ruminant Research 90: 219-226.

Gordon I, 2004. Reproductive Technologies in Farm Animals. Cabi Publishing: Cambridge, United Kingdom. 332 pp.

Greyling JP, 2000. Reproduction traits in the Boer goat doe. Small Ruminant Research 36 : 171-177.

Le Gal O et Planchenault D, 1993. Utilisation des races caprines exotiques dans les zones chaudes. Centre de coopération internationale en recherche agronomique pour le développement - ElevageÉlevage et Médecine Vétérinaire tropicale (CIRADEMVT), Paris, France. 261pp.

Ngona IA, 2015 Performances et facteurs d'influence de la reproduction caprine. Editions Universitaires Européennes, http://www.editions-ue.com/ $188 \mathrm{p}$.

Sakurai K, Ohkura S, Matsuyama S, Katoh K, Obara Y, Okamura H, 2004. Body growth and plasma concentration of metabolites and metabolic hormones during the pubertal period in female Shiba goats. Journal of Reproduction and Development 50: 197-205.

Webb EC and Mamabolo MJ, 2004. Production and reproduction characteristics of South African indigenous goats in communal farming systems. South African Journal of Animal Science, 34, supplement 1: 236-239. 
Zarazaga LA, Guzman JL, Dominguez C, Perez MC, Prieto $R, 2005$. Effect of plane of nutrition on seasonality of reproduction in Spanish Payoya goats. Animal Reproduction Science 87: 253267. 